\title{
Uso da Dinâmica Molecular na Descrição das Propriedades de Sistemas Líquidos
}

\author{
Gabriel R. Martins \& Ademir J. Camargo
}

O estudo da estrutura de solvatação molecular é de fundamental importância no entendimento da natureza microscópica da interação que ocorre entre soluto e solvente. O método de dinâmica molecular pode ser usado para descrever propriedades estáticas da estrutura de solvatação de um líquido através de ferramentas como a função de distribuição de pares e a distribuição do número de coordenação para cada camada solvatação que se forma entre o soluto e solvente. A trajetória gerada pela dinâmica molecular pode ajudar a estimar o coeficiente de difusão do líquido e o espectro de força (espectro vibracional) usando a função de autocorrelação da velocidade. Em geral, o solvente modifica os parâmetros geométricos e eletrônicos dos solutos, os quais são importantes na investigação de muitos fenômenos químicos que ocorrem em solução, sendo, portanto, de interesse em processos industriais e biotecnológicos.

Palavras-chave: dinâmica molecular; função de distribuição radial de pares; coeficiente de difusão e espectro de força.

The study of molecular salvation structure is of fundamental importance to understand the microscopic nature of the interaction that takes place between solute and solvent. The molecular dynamics method can be used to describe the static properties of the solvation structure of liquids using tools such as radial pair distribution function e coordination number distribution to each solvation shell formed between the solute and the solvent. The generated trajectory by molecular dynamics can help to estimate the liquid diffusion coefficient and the power spectra (vibrational spectra) using the velocity autocorrelation function. In general, the solvent modify the geometric and electronic parameters of the solutes, which are important in the investigation of many chemical phenomena that take place in solution, therefore, of interest in industrial and biotechnological process.

Keywords: molecular dynamics; radial pair distribution function; diffusion coefficient; power spectra. 


\section{Introdução}

Uma das principais ferramentas no estudo teórico do comportamento de meios nas fases líquida, gasosa e solida, é o método de simulação por dinâmica molecular (MD). ${ }^{1}$ Este método computacional avalia o comportamento dependente do tempo de um sistema molecular. Simulações de DM são capazes de fornecerem informações detalhadas sobre as flutuações e mudanças conformacionais de proteínas e ácidos nucléicos. ${ }^{2,3,4}$ Estes métodos são rotineiramente utilizados para investigar a estrutura, dinâmica e termodinâmica das moléculas de um sistema. Eles também vêm complementar e explicar muitos resultados obtidos a partir da determinação de estruturas por meio da cristalografia e de experimentos com RMN. A DM também tem fornecido grande contribuição ao planejamento de novos fármacos, ${ }^{5,6,7}$ por meio da simulação da interação entre o fármaco e o ambiente líquido no qual atuará.

Os métodos de simulação computacional são, sem dúvida, ferramentas poderosas para a compreensão dos fenômenos físicos e químicos da matéria. Tais métodos complementam e unem teoria e experimento. A simulação por DM permite-nos entender os experimentos e substituí-los quando os mesmos são muito perigosos ou que demandam elevados investimentos financeiros, podendo, inclusive, prever novos fenômenos. Existem dois modelos para a descrição do solvente em uma simulação por DM: o modelo contínuo e o modelo discreto. O modelo contínuo é um tipo de aproximação que considera o solvente de maneira implícita, onde este é baseado numa descrição contínua do meio. ${ }^{8}$ Já o modelo discreto trata o solvente explicitamente levando em consideração cada molécula que o compõe e as interações entre elas.

Em geral os métodos de simulação por DM podem ser divididos em dois grupos: os métodos de primeiros princípios ou ab initio (baseados em cálculos com constantes universais) e os métodos empíricos ou semiempíricos (baseados em parâmetros experimentais). ${ }^{9}$ Atécnica de Dinâmica Molecular Clássica frequentemente usa parâmetros empíricos, enquanto que as Dinâmicas Moleculares de Ehrenfest, ${ }^{10}$ Born-Oppenheimer ${ }^{11}$ e CarParrinello $^{12}$ usam teorias quânticas que não precisão recorrer à parâmetros experimentais. Como regra geral, uma descrição por primeiros princípios se faz necessária quando desejamos conhecer mais sobre a formação e quebra de ligações químicas, quando se tem a alteração de meios líquidos, quando a coordenação é variável etc. Se esses forem os casos, então fica evidente que uma simulação por primeiros princípios se faz necessária em detrimento a uma simulação por MD clássica. ${ }^{13}$

Uma simulação por DM envolve principalmente o estabelecimento das posições iniciais de cada átomo do sistema alvo de estudo, a escolha do ensemble onde será realizada a simulação, a introdução de condições periódicas de contorno a fim de se minimizar os problemas relacionados a descontinuidade do meio (modelo valido apenas para sistemas homogêneos), a movimentação atômica e a análise dos dados da simulação para as propriedades termodinâmicas, estruturais ou dinâmicas de interesse. Os sistemas comumente estudados em simulação de líquidos estão sob as condições do ensemble canônico, com temperatura $T$, volume $V$ e número $N$ de moléculas fixo (NVT), e o ensemble isobárico-isotérmico $(N p T)$, em que $p$ é a pressão do sistema. ${ }^{14}$

As forças sobre os átomos calculadas, na DM, são derivadas do potencial intermolecular, já o movimento dos átomos é obtido pelo uso de algum método de integração numérica, como o método de $\operatorname{Verlet}^{15}$ ou o método Velocity Verlet ${ }^{16}$. A DM se baseia, então, na resolução das equações de Newton para a movimentação dos átomos. As condições de partida são as posições de cada átomo e as suas velocidades (geradas a partir de números aleatórios e dimensionadas para a temperatura desejada). De posse das posições iniciais, velocidades e forças, é possível calcular as posições e velocidades dos átomos em um pequeno intervalo de tempo (um passo de tempo) adiante. A partir das novas posições, as forças são recalculadas e mais um passo de tempo é obtido. Este ciclo se repete durante toda a simulação.

A etapa final da simulação por DM é a análise das trajetórias do espaço de fase obtidas para a determinação das propriedades estruturais, dinâmicas e termodinâmicas (observáveis) do sistema. Vale lembrar que todas estas propriedades físico-químicas são obtidas após a relaxação do sistema. Durante a fase de relaxação, as propriedades do sistema não se mantêm constantes. Portanto, para equilibrar o sistema, deve-se deixar o mesmo evoluir por alguns milhares de passos, na temperatura especificada, 
antes de se iniciar a coleta das trajetórias do sistema que serão usadas no cálculo das propriedades desejadas.

Teoricamente, a Mecânica Estatística nos fornece os meios para descrever e predizer as propriedades macroscópicas da matéria a partir de seus constituintes microscópicos, enquanto que experimentalmente os princípios e métodos da Termodinâmica nos permitem correlacionar muitas das propriedades observáveis da matéria, tais como o coeficiente de difusão, calores específicos, calores de transformação etc. É de fundamental importância a necessidade das duas frentes de pesquisa, que se completam, para a evolução do conhecimento científico e tecnológico.

\section{Fundamentos Metodológicos}

\section{DISTRIBUIÇÃO RADIAL DE PARES E DISTRIBUIÇÃO DO NÚMERO DE COORDENAÇÃO}

Em uma simulação por DM, pode-se obter diversas informações sobre o arranjo estrutural. Isso pode ser feito através de uma função específica: a função distribuição radial, $g(r)$, que permite interpretações relativas à estrutura do sistema em estudo, tais como o número de camadas de solvatação ao redor de uma molécula e a existência de interações intermoleculares. De forma geral, pode-se dizer que ela relaciona números de átomos com distância, $r$, a um átomo de referência. ${ }^{17} \mathrm{~A}$ função distribuição radial de pares $g(r)$ entre os pares de átomos $i$ e $j$, é definida por

$$
g_{i j}(r)=\frac{N_{i j}(r, r+\Delta r)}{4 \pi r^{2} \Delta r \rho_{j}}
$$

onde $r$ é a distância entre os pares, $N \_i j(r, \Delta r)$ é o número médio (de todas as configurações consideradas de átomos da espécie $j$ encontrados em uma casca esférica de raio interno $r$ e raio externo $r+\Delta r$, centrada no átomo $i$, sendo que $4 \pi r^{2} \Delta r \rho_{j}$ corresponde ao volume da casca esférica; e $\rho_{j}$ é a densidade numérica da espécie $j$ na simulação.

A função de distribuição radial de pares é, portanto, a razão entre a probabilidade de se encontrar um átomo da espécie $j$ a certa distância radial do átomo $i$ e a probabilidade de se encontrar esse par de átomos em um sistema aleatoriamente distribuído com densidade similar. A Figura 1 mostra a função $g(r)$ e sua interpretação bidimensional. No centro do sistema, localiza-se o átomo de referência, seguido de duas camadas circulares. A primeira camada circular é composta por um número específico de átomos, representando, portanto, um pico correspondente no gráfico. A segunda camada é composta por outro número de átomos (maior que o primeiro), mas como sua área é maior do que a primeira, o pico é mais suave. ${ }^{18}$ Nota-se que a $g(r)$ é zero até o início da primeira camada, pois não existem átomos entre ela $\mathrm{e}$ $\mathrm{o}$ átomo de referência.

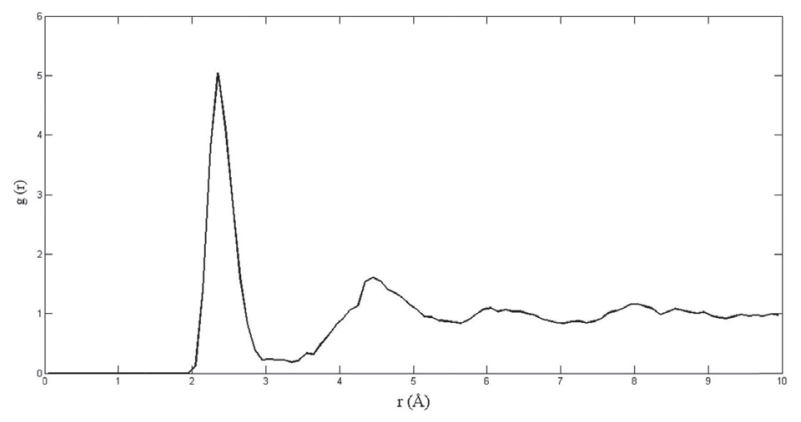

Figura 1. Representação bidimensional da distribuição radial de pares

À medida que a distância aumenta, a função se aproxima de 1 , pois a probabilidade de se encontrar um átomo no sistema estudado passa ser próxima à probabilidade de se encontrar um átomo em um sistema aleatoriamente distribuído. Em laboratório, as funções de distribuição radial de pares podem ser determinadas a partir de técnicas de difração de raios-X, espalhamento de nêutrons e difração de elétrons. ${ }^{19}$ Em uma simulação computacional, o cálculo da função distribuição radial de pares requer o cálculo das distâncias $r$ entre os átomos $i$ e $j$. O número de vezes que ocorre o par de átomos em função da distância $r$ é armazenado em um histograma. Esse histograma é incrementado ao longo da simulação, e por fim é normalizado para o número $N$ de moléculas utilizadas na caixa de simulação e para o número de passos usados no cálculo da distribuição radial de pares.

Os máximos das funções de distribuição radial de pares representam as distâncias médias entre vizinhos e, no caso especifico dos líquidos, estão associados às camadas de solvatação. ${ }^{14} \mathrm{~A}$ integração da Equação (1), até os mínimos correspondentes, fornece estimativas do 
número de vizinhos, ou seja, a distribuição do número de coordenação, $C_{i j}$. Por exemplo, para a primeira camada de solvatação com mínimo em $R_{c}$ temos,

$$
C_{i j}\left(R_{c}\right)=\int_{0}^{R_{c}} g_{i j}(r) \rho_{j} 4 \pi r^{2} d r
$$

onde $C_{i j}\left(R_{c}\right)$ é o número de primeiros vizinhos ao redor de um átomo ou molécula de interesse.

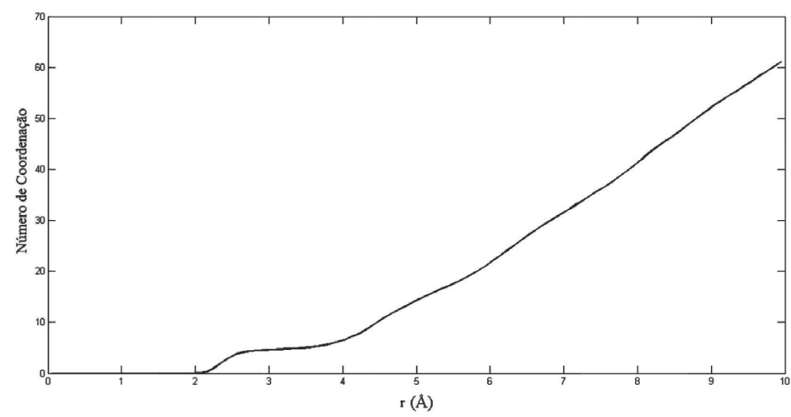

Figura 2. Representação do numero de coordenação. O patamar do gráfico em aproximadamente $2,5 \AA$ corresponde ao número de coordenação para a primeira esfera de solvatação.

Na Figura 2, temos a representação bidimensional da Distribuição do Número de Coordenação (DNC). O patamar localizado em $r$ aproximadamente 2,5 $\AA$ representa uma média para número de ligantes que se coordenam ao soluto durante toda a simulação. Este número de coordenação muitas vezes apresenta valores fracionários, isto se justifica quando levamos em consideração que o gráfico da Figura 2, é a sobreposição de vários gráficos (um para cada passo de tempo de simulação). Esta média representa a variação do número de coordenação para a camada de solvatação ao redor das moléculas de soluto em solução.

\section{OMOVIMENTOBROWNIANOE OCOEFICIENTE DE DIFUSÃO}

O Movimento Browniano (em homenagem ao seu descobridor em 1827, o botânico Robert Brown) é o deslocamento aleatório que as partículas inseridas em certo fluido estão sujeitas e que advêm do movimento térmico do sistema; ${ }^{20}$ já que a força aleatória que move estas partículas é devida à colisão com as demais partículas do fluido circundante. Estas colisões sucessivas, mesmo que no equilíbrio termodinâmico, mantém a agitação térmica do sistema e produzem certa resistência ao movimento, quando este é exercido por uma força externa. A difusão molecular é um exemplo de fenômeno de transporte de matéria onde um soluto é transportado devido aos movimentos das moléculas de um fluido. Estes movimentos fazem com que, do ponto de vista macroscópico, o soluto passe das zonas de altas concentrações para as zonas de baixas concentrações, como mostrado na Figura 3.

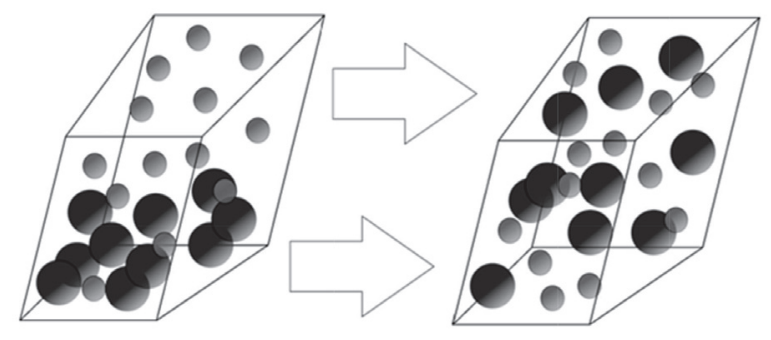

Figura 3. Na difusão, as moléculas do soluto se movimentam de um local mais concentrado para um local menos concentrado como indicado pelas setas.

A difusão é um processo natural por meio do qual um dado sistema, com determinadas condições iniciais, atinge o estado de equilíbrio após cada partícula seguir uma trajetória completamente aleatória. Existem duas leis para descrever o processo de difusão: a lei de Fick da difusão, a qual utiliza um coeficiente chamado de coeficiente de difusão. A outra, que não tem um nome formal, envolve um coeficiente de transferência de massa. A Figura 4 mostra a representação bidimensional da difusão de uma partícula em um fluido.

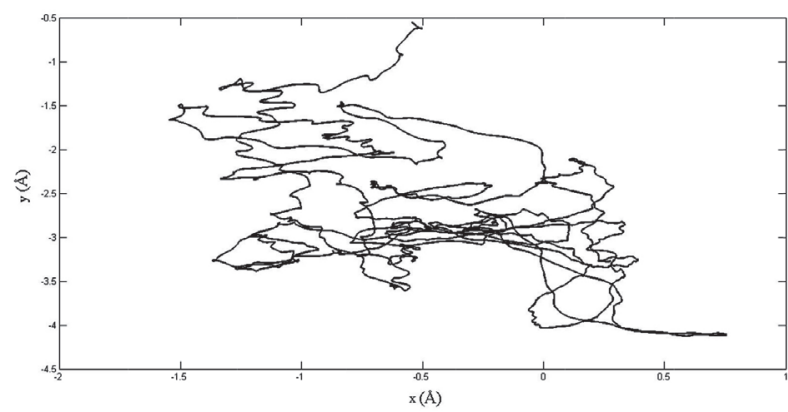

Figura 4. Representação esquemática bidimensional da trajetória de uma partícula em um meio líquido. O traçado caótico é previsto pelo movimento browniano. 
A lei de Fick afirma que o fluxo $\mathbf{j}$ de uma espécie que está difundindo é proporcional ao gradiente da concentração desta espécie, ou seja,

$$
\mathrm{j}=-D \nabla_{\mathbf{r}} c(\mathbf{r}, t)
$$

onde a constante de proporcionalidade $D$ é chamada de coeficiente de difusão e $c$ é a função que dá a concentração da espécie no ponto $\mathbf{r}$ e tempo $t$. Quando uma mesma espécie difunde nela mesma, chamamos este processo de autodifusão. Para computar o perfil da concentração de um soluto, supõe-se que no instante $t$ $=0$ todo o soluto se encontra concentrado na origem do sistema de coordenadas. Para calcularmos o perfil da evolução temporal da concentração do soluto, precisamos combinar a lei de Fick com a lei da conservação das massas, dada por

$$
\frac{\partial c(\mathrm{r}, t)}{\partial t}+\nabla_{r} \cdot \boldsymbol{j}(\mathrm{r}, t)=0
$$

Inserindo (3) em (4), obtemos

$$
\frac{\partial c(\mathrm{r}, t)}{\partial t}-D \nabla_{r}^{2} c(\mathrm{r}, t)=0 .
$$

Para calcular o coeficiente de difusão não precisaremos resolver (5) para encontrar $c(\mathbf{r}, t)$. É suficiente termos a dependência temporal do segundo momento de $c(\mathbf{r}, t)$, ou seja,

$$
\left\langle\mathbf{r}^{2}(t)\right\rangle \equiv \int \mathbf{r}^{2} c(\mathbf{r}, t) d \mathbf{r}
$$

onde impomos o fato de que

$$
\int c(\mathbf{r}, t) d \mathbf{r}=1 .
$$

Podemos obter uma equação para a evolução temporal de $\left\langle\mathbf{r}^{2}(t)\right\rangle$, multiplicando a Equação (5) por $\mathbf{r}^{2} \mathrm{e}$ integrando sobre todo o espaço, ou seja,

$$
\begin{gathered}
\mathbf{r}^{2} \frac{\partial}{\partial t} c(\mathbf{r}, t)=\mathbf{r}^{2} D \nabla_{\mathbf{r}}^{2} c(\mathbf{r}, t), \\
\frac{\partial}{\partial t} \int \mathrm{r}^{2} c(\mathrm{r}, t) d \mathbf{r}=D \int \mathrm{r}^{2} \nabla_{\mathrm{r}}^{2} c(\mathrm{r}, t) d v
\end{gathered}
$$

Usando (6) do lado esquerdo de (9) e integrando o lado direito de (9) por partes, obtemos

$$
\frac{\partial\left\langle\mathrm{r}^{2}(t)\right\rangle}{\partial t}=2 d D
$$

onde ${ }^{D}=\frac{1}{2 d} \frac{\partial\left\langle\mathrm{r}^{2}(t)\right\rangle}{\partial t}$ e $d$ representa a dimensionalidade do sistema. A Equação (10) foi derivada inicialmente por Einstein. ${ }^{21}$ Enquanto, $D$, é o coeficiente de transporte macroscópico, $\left\langle\mathbf{r}^{2}(t)\right\rangle$ tem uma interpretação microscópica, isto é, $\left\langle\mathbf{r}^{2}(t)\right\rangle$ representa o deslocamento médio quadrático que a molécula realiza em um intervalo de tempo $t$. Isto sugere imediatamente um procedimento para medirmos o coeficiente de difusão usando a trajetória obtida pela simulação computacional de DM. Para cada partícula $i$, medimos a distância deslocada no tempo $t, \Delta \mathbf{r}_{i}(t)$, e construímos um gráfico do deslocamento médio quadrático em função do tempo $t$ :

$$
\left\langle(\Delta \mathbf{r}(t))^{2}\right\rangle=\frac{1}{N} \sum_{i=1}^{N}\left(\Delta \mathbf{r}_{i}(t)\right)^{2},
$$

onde $N$ representa o número total de partículas do soluto, $\left\langle(\Delta \mathbf{r}(t))^{2}\right\rangle$ é o deslocamento médio quadrático, $\left(\Delta \mathbf{r}_{i}(t)\right)^{2}$ é o deslocamento quadrático da partícula $i$. De acordo com a Equação (10), para obter o coeficiente de difusão $D$ basta ajustar uma reta, por mínimos quadrados, aos pontos do gráfico, como é mostrado na Figura 5, calcular o coeficiente angular da reta e dividir por $2 d$, onde $d=3$ se estivermos trabalhando em três dimensões.

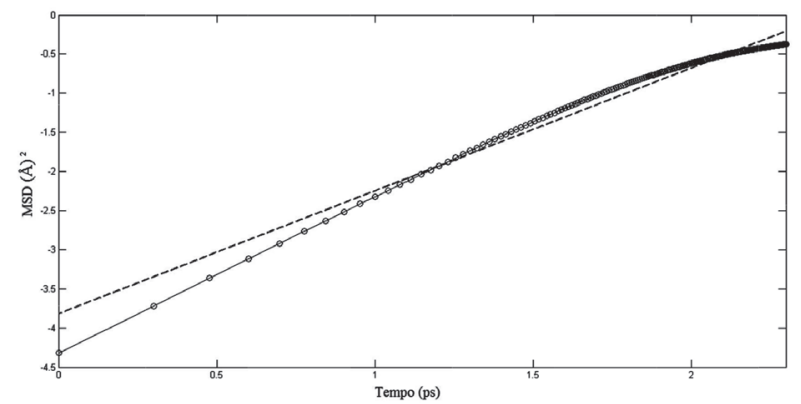

Figura 5. Deslocamento médio quadrático para uma partícula em solução (linha contínua) e reta ajustada por mínimos quadrados (linha tracejada).

Sendo o número de passos de tempo da simulação, $\theta$, pode-se mostrar que no limite de tempos muito grandes, $\theta \rightarrow \infty$, de acordo com o teorema do limite central, a distribuição de percursos da partícula tende a uma função gaussiana. Com as contribuições de Green- 
Kubo, ${ }^{22}$ o coeficiente de difusão é calculado na prática pela seguinte equação:

$$
6 t D=\left\langle\left|\mathbf{r}_{i}(t)-\mathbf{r}_{i}(0)\right|^{2}\right\rangle,
$$

a equação (12) contém o autotermo de deslocamento de cada partícula $i$ do sistema num dado tempo $t$. Os brackets denotam a média no ensemble, que em DM significa uma média sobre todas as partículas $i$ e origens no tempo.

\section{FUNÇÕES DE AUTOCORRELAÇÃO DA VELOCIDADE E OS ESPECTROS DE ABSORÇÃO}

A Função de Autocorrelação da Velocidade (VACF) é um típico exemplo de uma função de correlação que depende do tempo da simulação da DM. Além de fornecer dados importantes sobre a natureza dos processos dinâmicos que ocorrem em um sistema molecular, a VACF também ajuda na elaboração do espectro de força do líquido simulado.

As VACFs tendem assitoticamente a zero, significando que ao longo do tempo as moléculas vão perdendo a "informação" das suas velocidades iniciais e a VACF passa então a ter o comportamento mostrado na Figura 6. Os trabalhos de Alder e Wainwright ${ }^{23}$ mostraram que existe um fenômeno chamado efeito vórtice, ou seja, um escoamento giratório onde as linhas de corrente apresentam um padrão circular ou espiral. Neste tipo de fenômeno o movimento de uma molécula através do líquido cria à sua volta uma corrente retardada na direção da velocidade inicial. ${ }^{24}$

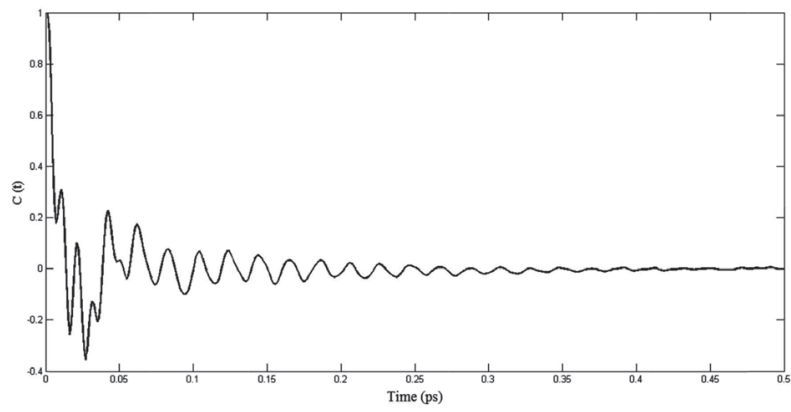

Figura 6. Represetação da Função de Autocorrelação da Velocidade (VACF)

O cálculo da função de autocorrelação da velocidade envolve o produto escalar das velocidades antes e depois de um passo de tempo, o seja se tormarmos a origem do movimento como tendo tempo $t=t_{0}$, o próximo passo então terá o tempo $t=t_{0}+\Delta t$, onde, $\Delta t$, será o acrescimo ao tempo inical, $t_{0}$. A função de autocorrelação da velocidade para cada átomo do sistema estudado será dada então por

$$
C(t=\Delta t)=\frac{1}{N} \sum_{i=1}^{N}\left(v_{i}\left(t=t_{0}\right) \cdot v_{i}\left(t=t_{0}+\Delta t\right)\right) .
$$

Podemos repetir este raciocínio para uma trajetoria grande de varios intervalos de tempo totalizando um numero total de intervalos, dessa forma obteremos uma sequência de pontos para a função de autocorrelação da velocidade:

$$
C(t=n \Delta t)=\frac{1}{N} \sum_{i=1}^{N}\left(v_{i}\left(t=t_{0}\right) \cdot v_{i}\left(t=t_{0}+n \Delta t\right)\right)
$$

ou de forma reduzida,

$$
C(t)=\left\langle v_{i}(0) \cdot v_{i}(t)\right.
$$

O movimento dos átomos é oscilatório, ou seja, o átomo vibra para frente e para trás, invertendo a sua velocidade no final de cada oscilação. Se calcularmos a VACF, obteremos uma função que oscila fortemente de positivo para valores negativos e vice-versa. As oscilações não serão de igual magnitude e decairão com o tempo, porque ainda há forças perturbativas atuando sobre cada átomo do sistema de modo a criar um movimento oscilatório. Então, o que vemos é uma função semelhante a um movimento harmônico amortecido como mostrado na Figura 6. Usando a Equação (10) podermos escrever a relação de Green-Kubo agora com a inserção da função de autocorrelação da velocidade, ou seja,

$$
D=\frac{1}{2 d} \int_{t=0}^{t=\infty}\left\langle v_{i}(0) \cdot v_{i}(t)\right\rangle d t,
$$

onde $D=\frac{1}{2 d} \int_{t=0}^{t=\infty} C(t) d t$.

As intensidades do espectro de força, $i(\omega)$, para o líquido podem ser calculadas tomando-se a transformada de Fourier da função de autocorrelação da velocidade, $C(t)$, que transfere a informação sobre as correlações ao longo da trajetória da dinâmica molecular para as frequências $\omega$, obtidas em cada passo de tempo da simulação, como mostra a relação 


$$
I(\omega)=\int_{-\infty}^{\infty} \exp (-2 \pi i \omega t) C(t) d t
$$

\section{Conclusões}

$\mathrm{O}$ estudo de sistemas líquidos usando a DM vem ganhando cada vez mais importância nos laboratórios de pesquisa ao redor do mundo. ${ }^{25} \mathrm{~A}$ alta velocidade de crescimento desta área de pesquisa tem sido possível devido ao fato de que as simulações computacionais permitem diminuir a interface entre teoria e experimento. A simulação de DM tem sido usada pelos pesquisadores como uma poderosa ferramenta orientadora no planejamento de experimentos e/ou queimar etapas desnecessárias durante a realização de experimentos que envolva, por exemplo, uma reação química em condições de temperatura e pressão específicas. A DM pode ser usada para obter importantes informações sobre a natureza de um sistema líquido. Pode ser usada na obtenção de informações da estrutura de solvatação do solvente ao redor do soluto; calcular o número de coordenação entre soluto e solvente; estimar o coeficiente de difusão do soluto em um líquido e até mesmo fazer previsão do espectro de força (espectro vibracional) do sistema. Enfim, cabe a nós químicos analisar cada propriedade, cada sistema e cada processo, com a devida cautela e com as ferramentas corretas.

\section{Referências Bibliográficas}

1. Haile, J. M.; Molecular Dynamics Simulation: Elementary Methods, Wiley-Interscience: New York, 1997.

2. Hansson, T.; Oostenbrink, C.; van Gunsteren, W.; Curr. Opin Struct. Biol. 2002, 12, 190.

3. Karplus, M.; McCammon, J. A.; Nat. Struct. Biol. 2002, 9, 646.

4. Norberg, J.; Nilsson, L.; Quart. Rev. Biophys. 2003, 36, 257.

5. Alonso, H.; Bliznyuk, A. A.; Gready, J. E.; Med. Res. Rev. 2006, 26,531 .

6. Adcock, S. A.; McCammon, J. A.; Chem. Rev. 2006, 106, 1589.

7. Rognan, D.; Perspect. Drug Discov. Des., 1998, 9/10/11, 181.

8. Pliego Jr., J. R.; Quím. Nova. 2006, 29, 535.

9. Morgon, N. M.; Coutinho, K.; \& Colaboradores; Métodos de Química Teórica e Modelagem Molecular, Editora Livraria da Física: São Paulo, 2000.

10. Xiaosong, L.; Tully, J. C.; Schlegel, H. B.; Frisch, M. J.; J. Chem. Phys. 2005, 123, 084106.
11. Schlegel, H. B.; Bul. Kor. Chem. Soc. 2003, 24, 837.

12. Car, R.; Parrinello, M.; Phys. Rev. Lett. 1985, 55, 2471.

13. Kohanoff, J.; Electronic Structure Calculations for Solids and Molecules: Theory and Computational Methods, Cambridge University Press: Cambridge, 2006.

14. Barlette, V. E.; Freitas, L. C. G.; Quím. Nova. 1999, 22, 254.

15. Verlet, L.; Phys. Rev. 1967, 159, 98.

16. Swope, W. C.; Andersen, H. C.; Berens, P. H.; Wilson, K. R.; J. Chem. Phys. 1982, 76, 637.

17. Rapaport, D. C.; The Art of Molecular Dynamics Simulation, Cambridge University Press:Cambridge, 2004.

18. Allen, M. P.; Tildesley, D. J. Computer Simulation of Liquids, Oxford University Press: New York, 1989.

19. Teixeira, J.; Molecular Liquids: New Perspectives in Physics and Chemistry, Kluwer Academic Publisher: Dordrecht, 1992.

20. Mazo, R. M.; Brownian Motion: Fluctuations, Dynamics, and Applications, Oxford University Press: New York, 2009.

21. Salinas, S.; Rev. Bras. Ens. Fís., 2005, 27, 263.

22. Kubo, R.; Science, 1986, 233, 330.

23. Alder, B. J.; Wainwright, T. E.; Physical Review A, 1970, 1, 18.

24. Fernandes, F. M. S. S.; Química. 2004, boletim nº 63 da SPQ, 49.

25. Hutter, J.; Marx, D. Ab Initio Molecular Dynamics: Basic Theory and Advanced Methods. Cambridge University Press: Cambridge, 2009.

\section{Gabriel R. Martins* \& Ademir J. Camargo}

${ }^{1}$ Unidade Universitária de Ciências Exatas e Tecnológicas, Universidade Estadual de Goiás, Campus Henrique Santillo, BR 153, Km 98, CEP 75001-970, Anápolis, GO, Brasil.

*e-mail: gabrielrodriguesm@yahoo.com.br 